$$
T_{n}(z) \sim n !\left(\frac{e^{z}}{n z}\right)^{n} \phi_{J}(z)-\sum_{r=0}^{\infty}\left(\frac{1}{n}\right)^{r} U_{r}(z) .
$$

If $z=-1$, (5) reduces to Copson's series. Ramanujan's approximation for $S_{n}(1)$ is, in view of (5), a considerably more singular result than it would otherwise appear.

\title{
REFERENCES
}

1. E. T. Copson, An approximation connected with $e^{-x}$, Proc. Edinburgh Math. Soc. (2) 3 (1932-33), 201-206.

2. S. Ramanujan, Collected papers, p. 26, Cambridge, 1927.

3. G. Szegö, Über einige von S. Ramanujan gestellte Aufgaben, J. London Math. Soc. 3 (1928), 225-232.

4. G. N. Watson, Approximations connected with $e^{x}$, Proc. London Math. Soc. (2) 29 (1928-29), 293-308.

University of North Carolina

\section{ON THE LOCAL LINEARIZATION OF DIFFERENTIAL EQUATIONS ${ }^{1}$}

\section{PHILIP HARTMAN}

1. Consider the autonomous system of real, nonlinear differential equations

(1.1) $x^{\prime}=E x+F(x)$, where $F(|x|)=o(|x|)$ as $x \rightarrow 0$,

$x$ is a (Euclidean) vector, $F(x)$ a smooth vector-valued function of $x$, and $E$ a constant matrix with eigenvalues $e_{1}, e_{2}, \cdots$ satisfying

$$
\operatorname{Re} e_{j} \neq 0 \text {. }
$$

Let the solution $\xi(t, x)$ of (1.1) starting at $x$ for $t=0$ be written as

$$
T^{t}: x^{t}=\xi(t, x)=e^{E t} x+X(t, x),
$$

where $X(t, x)=o(|x|)$ as $x \rightarrow 0$ (for fixed $t$ ). Thus if $T^{t}$ is considered as a map $x \rightarrow x^{t}$, for fixed $t$, the composition rule

$$
T^{t} T^{s}=T^{t+8}
$$

is valid for small $|x|$. Correspondingly, the linear system

Received by the editors May 26, 1962.

${ }_{1}^{1}$ This research was supported by the Air Force Office of Scientific Research. 


$$
u^{\prime}=E u
$$

leads to the linear maps

$$
L^{t}: u^{t}=e^{E t} u
$$

The object of this note is to prove

$\left(^{*}\right)$ In (1.1), let $F(x)$ be of class $C^{\prime}$ for small $|x|$ (or uniformly Lipschitz continuous with a Lipschitz constant for $|x| \leqq \epsilon$ which tends to 0 as $\epsilon \rightarrow 0)$ and let the eigenvalues of $E$ satisfy (1.2). Let $x(t)=\xi(t, x)$ in (1.3) be the solution of (1.1) satisfying $x(0)=x$. Then there exists a topological map

$$
R: u=u(x)
$$

of a neighborhood of $x=0$ onto a neighborhood of $u=0$ such that

$$
R T^{t} R^{-1}=L^{t}
$$

The analogue of this result was proved in [2] under the assumption that $F$ is of class $C^{2}$. The proof here will be simpler than that of [2] by avoiding the use of Theorem (II) of [1] and by following the procedure of Sternberg [3, p. 817] for passing from the linearization of $T^{1}$ to that of $T^{t}$. This is accomplished by carrying out some of the normalizations, used in [2] for $T^{1}$, for the entire "group" $T^{t}$ and by an examination of the stable and unstable manifolds of $T^{t}$.

The proof will be given only for the case that $F$ is of class $C^{1}$; the arguments for the case that $F$ is uniformly Lipschitz continuous are analogous.

2. It will be supposed that not all $e_{j}$ satisfy $\operatorname{Re} e_{j}>0$ or $\operatorname{Re} e_{j}<0$, as this case is simple (cf. [2]). After a suitable linear change of variables, it can be supposed that $E$ is in a suitable normal form (to be specified by (2.3) and (2.4), below).

Since the assumption and assertion of $\left({ }^{*}\right)$ are local, nothing is changed if $F(x)$ is altered outside of a small vicinity of $x=0$. Thus there is no loss of generality in supposing that if $\theta>0$ is arbitrary, then there is an $s=s_{\theta}>0$ such that $s_{\theta} \rightarrow 0$ as $\theta \rightarrow 0, F(x)$ is defined for all $x, F(x)=0$ for $|x| \geqq s$, and that the norm $\left|F_{x}\right|$ of the Jacobian matrix $F_{x}$ does not exceed $\theta$ for all $x$. For otherwise, $F(x)$ can be replaced by $F(x) \phi(|x|)$, where $\phi(t)$ is a smooth function for $t \geqq 0$ satisfying $\phi(t)=1$ for $0 \leqq t \leqq s / 2, \phi(t)=0$ for $t \geqq s,|d \phi / d t| \leqq 3 / s$ and $s>0$ is sufficiently small (cf. [2, p. 612]). Thus $T^{t} x$ is defined for all $x$ and $-\infty<t<\infty$ and is indeed a group of maps.

It follows from (1.1) and $F(x)=0$ for $|x| \geqq s$ that there exists an 
$r=r_{\theta}>0$ such that $r_{\theta} \rightarrow 0$ as $\theta \rightarrow 0$ and the general solution (1.3) of (1.1) satisfies

$$
x^{t}=e^{E t} x \quad \text { if }|x| \geqq r, 0 \leqq t \leqq 1 .
$$

In fact, $r>0$ can be any number such that a solution of (1.1) beginning for $t=0$ at $x,|x| \geqq r$, does not enter $|x| \leqq s$ for $0 \leqq t \leqq 1$.

There also exists an $\epsilon=\epsilon_{\theta}$ such that $\epsilon_{\theta} \rightarrow 0$ as $\theta \rightarrow 0$ and the Jacobian matrix $X_{x}$ of $X(t, x)$ in (1.3) satisfies

$$
\left|X_{x}(t, x)\right| \leqq \epsilon \quad \text { for all } x \text { and } 0 \leqq t \leqq 1 .
$$

In view of (1.3) and (2.1), it is sufficient to verify (2.2) only for $|x| \leqq r$, where $x^{t}=\xi(t, x)$ is uniformly bounded for $0 \leqq t \leqq 1$. The Jacobian matrix $\partial x^{t} / \partial x=e^{E t}+X_{x}$ satisfies a linear differential equation and the variation of constants $\partial x^{t} / \partial x=e^{E t} J$ defines a matrix $J=J(t, x)$ satisfying the differential equation $J^{\prime}=e^{-E t} F_{x}\left(x^{t}\right) e^{E t} J$ and the initial condition $J=I$ at $t=0$, where $I$ is the unit matrix. It is clear that $J$ is uniformly bounded for $|x| \leqq r, 0 \leqq t \leqq 1$ (and $0<\theta \leqq 1$ ). Since $\left|F_{x}\right| \leqq \theta$, there is a constant $c$ such that $\left|J^{\prime}\right| \leqq c \theta$ for $0 \leqq t \leqq 1$ and so $|J(t, x)-I| \leqq c \theta$ for $0 \leqq t \leqq 1$. Hence $\epsilon=\epsilon_{\theta}$ can be chosen to be $c \theta$ sup $\left|e^{E t}\right|, 0 \leqq t \leqq 1$.

By assuming that $E$ is in a suitable normal form and writing $(x, y)$ in place of $x,(X(t, x, y), Y(t, x, y))$ in place of $X(t, x)$, etc., (1.3) can be written in the form

$$
T^{t}: x^{t}=e^{\Gamma t} x+X(t, x, y), \quad y^{t}=e^{\Delta t} y+Y(t, x, y),
$$

where $x, y$ are vectors; $\Gamma, \Delta$ matrices with eigenvalues satisfying $\operatorname{Re} \gamma_{j}<0, \operatorname{Re} \delta_{k}>0$, respectively; and $X, Y$ are of class $C^{1}$ for all $x, y$ and $t$. It can also be supposed that there are numbers $a, b$ such that

$$
\left|e^{\Gamma}\right| \leqq a<1 \text { and }\left|e^{-\Delta}\right| \leqq 1 / b<1 \text {. }
$$

It is clear from (1.3) that

$$
X, Y=o(|x|+|y|) \text { as } x, y \rightarrow 0 \text { (for fixed } t \text { ) }
$$

and from (2.1), (2.2) that

$$
\begin{aligned}
& \text { (2.6) } \quad X, Y=0 \quad \text { for }|x|^{2}+|y|^{2} \geqq r^{2} \text { and } 0 \leqq t \leqq 1 \\
& \text { (2.7) }\left|X_{x}\right|,\left|X_{y}\right|,\left|Y_{x}\right|,\left|Y_{y}\right| \leqq \epsilon \text { for } 0 \leqq t \leqq 1 \text { and all } x, y \text {. }
\end{aligned}
$$

Corresponding to the form (2.3) of (1.3), the linear map (1.6) has the form

$$
L^{t}: u^{t}=e^{\Gamma t} u, \quad v^{t}=e^{\Delta t} v .
$$

3. Let $M_{ \pm}$be the set of points $\left\{(x, y): T^{t}(x, y) \rightarrow(0,0)\right.$ as $\left.t \rightarrow \pm \infty\right\}$. 
Then, as is known, the subsets of $M_{ \pm}$in a sufficiently small vicinity of $x=y=0$, are manifolds of class $C^{1}$ of the respective forms $y=\eta_{0}(x)$, $x=\xi_{0}(y)$. The proof of this (local) assertion in [1, §7] can be adapted to show that if $r, \epsilon$ are sufficiently small in (2.6)-(2.7), then, under the normalizations (2.3) $-(2.7)$, the sets $M_{ \pm}$are $C^{1}$ manifolds of the forms $y=\eta_{0}(x), x=\xi_{0}(y)$, defined for all $x, y$, respectively, with $\xi_{0}(0)=0$, $\xi_{0 y}(0)=0, \eta_{0}(0)=0, \eta_{0 y}(0)=0$; also $\xi_{0}, \eta_{0}$ and their Jacobian matrices have a bound, say $\delta$, for all $x, y$, such that $\delta \rightarrow 0$ as $\epsilon, r \rightarrow 0$.

It is clear that if $\delta$ is small enough, then

$$
M_{+} \cap M_{-}=(0,0) .
$$

It will also be verified that

$$
\begin{aligned}
& (x, y) \notin M_{+} \Rightarrow\left|x^{n}\right| \rightarrow 0,\left|y^{n}\right| \rightarrow \infty \text { as } n \rightarrow \infty, \\
& (x, y) \notin M_{-} \Rightarrow\left|x^{n}\right| \rightarrow \infty,\left|y^{n}\right| \rightarrow 0 \text { as } n \rightarrow-\infty .
\end{aligned}
$$

In order to obtain (3.2), consider the maps

$$
R_{0}: u=x, v=y-\eta_{0}(x) ; \quad R_{0}^{-1}: x=u, y=v+\eta_{0}(u)
$$

and

$$
R_{0} T^{1} R_{0}^{-1}: u^{1}=e^{\Gamma} u+U_{0}(u, v), \quad v^{1}=e^{\Delta} v+V_{0}(u, v),
$$

where $U_{0}, V_{0}$ and their Jacobians are small for all $(u, v)$. In particular let $\left|V_{0 v}\right| \leqq \delta_{0}$, where $\delta_{0} \rightarrow 0$ as $\epsilon, r \rightarrow 0$. In $(u, v)$-coordinates, $M_{+}: v=0$ is an invariant manifold so that $V_{0}(u, 0)=0$. Thus $\left|V_{0}(u, v)\right| \leqq \delta_{0}|v|$ and $\left|v^{1}\right| \geqq\left|e^{\Delta_{v}}\right|-\delta_{0}|v| \geqq\left(b-\delta_{0}\right)|v|$, by (2.4). Hence $\left|v^{n}\right| \geqq\left(b-\delta_{0}\right)^{n}|v|$, where $b-\delta_{0}>1$ if $\delta_{0}$ is sufficiently small. If $(x, y) \notin M_{+}$and $(u, v)$ is given by (3.4), then $v \neq 0$ and $\left|v^{n}\right| \rightarrow \infty$ as $n \rightarrow \infty$. The $R_{0}^{-1}$ image of $\left(u^{n}, v^{n}\right)$ is $\left(x^{n}, y^{n}\right)$ and $y^{n}=v^{n}+\eta_{0}\left(u^{n}\right)$. The boundedness of $\eta_{0}(u)$ gives $\left|y^{n}\right| \rightarrow \infty$ as $n \rightarrow \infty$. For large $n,(2.6)$ and (2.3) show that $\left|x^{n}\right|=\left|e^{\Gamma} x^{n-1}\right| \leqq a\left|x^{n-1}\right|$. Hence $\left|x^{n}\right| \rightarrow 0$ as $n \rightarrow \infty$. This gives the relations (3.2). The relations (3.3) are verified in the same way.

4. Consider the problem of linearizing $T^{1}$, that is, the problem of the existence of a continuous, one-to-one map of the $(x, y)$-space onto the $(u, v)$-space

$$
R_{1}: u=U_{1}(x, y), \quad v=V_{1}(x, y)
$$

such that

$$
L^{1} R_{1}=R_{1} T^{1} .
$$

The existence of such an $R_{1}$ was proved in [2] under the additional normalizations $X(1,0, y)=0, Y(1, x, 0)=0$ (i.e., $M_{+}: y=0$ and 
$\left.M_{-}: x=0\right)$. But these normalizations are inessential.

The relation (4.2) is equivalent to a functional equation

$$
e^{\Delta} V_{1}(x, y)=V_{1}\left(e^{\Gamma} x+X(1, x, y), e^{\Delta} y+Y(1, x, y)\right)
$$

for $V_{1}$ and a similar one for $U_{1}$. In order to obtain a solution for $V_{1}$, consider the successive approximations

(4.40) $V^{0}(x, y)=y$,

$\left(4.4_{n}\right) V_{n}(x, y)=e^{-\Delta} V^{n-1}\left(e^{\Gamma} x+X(1, x, y), e^{\Delta} y+Y(1, x, y)\right)$ for $n \geqq 1$.

If $r, \epsilon$ in (2.6), (2.7) are sufficiently small, a simple induction shows the existence of $K, \delta, \eta$, with $0<\delta, \eta<1$, such that $\left|V^{n}-V^{n-1}\right|$ $\leqq K \eta^{n}(|x|+|y|)^{\delta}$ for $n=1,2, \cdots ;\left[2\right.$, p. 613]. Hence $V_{1}(x, y)$ $=\lim V^{n}(x, y), n \rightarrow \infty$, exists uniformly on bounded $(x, y)$-sets. The existence of a continuous $U_{1}(x, y)$ is obtained similarly.

It follows that there is a continuous map (4.1) satisfying (4.2). It therefore only remains to show that $R_{1}$ is one-to-one and onto the $(u, v)$-space. Note that from $(2.4),(2.6),\left(4.4_{n}\right)$ and corresponding formulae for the successive approximations of $U_{1}$, it is seen that

(4.5) $\quad R_{1}(0,0)=(0,0)$,

(4.6) $U_{1}(x, y)=x$ if $|x| \geqq r / a$ and $V_{1}(x, y)=y$ if $|y| \geqq r$.

Suppose, if possible, that there exists a pair of points $\left(x_{1}, y_{1}\right),\left(x_{2}, y_{2}\right)$ satisfying

$$
R_{1}\left(x_{1}, y_{1}\right)=R_{1}\left(x_{2}, y_{2}\right) \text { but }\left(x_{1}, y_{1}\right) \neq\left(x_{2}, y_{2}\right) .
$$

It follows from (4.2) and a simple induction that

$$
R_{1}\left(x_{1}^{n}, y_{1}^{n}\right)=R_{1}\left(x_{2}^{n}, y_{2}^{n}\right) \quad \text { for } n=0, \pm 1, \cdots,
$$

where $\left(x_{j}^{n}, y_{j}^{n}\right)=T^{n}\left(x_{j}, y_{j}\right)$. Since $T^{t}$ is one-to-one by (2.3) and (2.7),

$$
\left(x_{1}^{n}, y_{1}^{n}\right) \neq\left(x_{2}^{n}, y_{2}^{n}\right) \quad \text { for } n=0, \pm 1, \cdots .
$$

Consider first the case (i) that either $\left(x_{1}, y_{1}\right)$ or $\left(x_{2}, y_{2}\right)$ is on an unstable or stable manifold $M_{ \pm}$, say, $\left(x_{1}, y_{1}\right) \in M_{+}$. Then $\left(x_{2}, y_{2}\right) \in M_{+}$. For otherwise, $\left(x_{1}^{n}, y_{1}^{n}\right) \rightarrow(0,0)$ and, by (3.2), $\left|y_{2}^{n}\right| \rightarrow \infty$, as $n \rightarrow \infty$, so that $R_{1}\left(x_{1}^{n}, y_{1}^{n}\right) \rightarrow 0$, by (4.5), and $\left|R_{1}\left(x_{2}^{n}, y_{2}^{n}\right)\right| \rightarrow \infty$, by (4.6), as $n \rightarrow \infty$. This contradicts (4.8). Now $\left(x_{j}, y_{j}\right) \in M_{+}$for $j=1,2$ implies that $y_{j}^{n}=\eta_{0}\left(x_{j}^{n}\right)$ for $j=1,2$, where $M_{+}: y=\eta_{0}(x)$. It follows from (3.1), (3.3) that $\left|x_{j}^{n}\right| \rightarrow \infty$ as $n \rightarrow-\infty$. But then (4.6) and (4.8) imply that $x_{1}^{n}=x_{2}^{n}$ if $-n$ is large. Hence $y_{1}^{n}=\eta_{0}\left(x_{1}^{n}\right)$ is the same as $y_{2}^{n}=\eta_{0}\left(x_{2}^{n}\right)$ for large $-n$. This contradicts (4.9)and shows that case (i) cannot occur. 
Consider the case (ii) that neither $\left(x_{1}, y_{1}\right)$ nor $\left(x_{2}, y_{2}\right)$ is on $M_{ \pm}$. Then $\left|x_{j}^{n}\right| \rightarrow 0,\left|y_{j}^{n}\right| \rightarrow \infty$ as $n \rightarrow \infty$ and $\left|x_{j}^{n}\right| \rightarrow \infty,\left|y_{j}^{n}\right| \rightarrow 0$ as $n \rightarrow-\infty$ for $j=1,2$ (cf. (3.2)-(3.3)). The argument in [2, p. 614] shows that case (ii) is impossible if $a, b, \epsilon$ in (2.4), (2.7) satisfy $a+2 \epsilon<1<b-2 \epsilon$. Hence $R_{1}$ is one-to-one. It is an open map by invariance of domain. It is also easy to see from (4.6) that the range of $R$ is closed. Hence $R$ is onto.

5. It was observed by Sternberg [3, p. 817] that (4.2) implies that the map

$$
R=\int_{0}^{1} L^{-t} R_{1} T^{t} d t
$$

satisfies, for all $t$,

$$
L^{t} R=R T^{t} .
$$

Thus, in order to complete the proof of $\left({ }^{*}\right)$, i.e., of $(1.8)$, it only remains to verify that the map (5.1) is one-to-one and onto.

In view of the normalizations above for $T^{t}, 0 \leqq t \leqq 1$, this verification turns out to be the same as the verification that $R_{1}$ is one-to-one. In fact, if (5.1) is written as

$$
R: u=U(x, y), \quad v=V(x, y),
$$

then

$$
R(0,0)=(0,0) .
$$

Also, by (5.1) and (2.3), (2.6), (4.8), there is a constant $c>0$ such that

$$
U(x, y)=x \text { if }|x| \geqq c, \quad V(x, y)=y \text { if }|y| \geqq c .
$$

Thus, analogues of the relations (4.2), (4.5), (4.6) which were used to show that $R_{1}$ is one-to-one hold for $R$, namely, (5.2), (5.4), (5.5). It follows that $R$ is one-to-one and onto. This completes the proof of $(*)$.

\section{REFERENCES}

1. P. Hartman, On local homeomorphisms of Euclidean spaces, Bol. Soc. Mat. Mexicana 5 (1960), 220-241; Symposium Internacional de Ecuaciones Diferenciales Ordinaries, Universidad Nac. Autónoma Mexico y Soc. Mat. Mexicana, 1961.

2. - A lemma in the theory of structural stability of differential equations, Proc. Amer. Math. Soc. 11 (1960), 610-622.

3. S. Sternberg, Local contractions and a theorem of Poincare, Amer. J. Math. 79 (1957), 809-824.

\section{The Johns Hopkins University}

